\title{
Heritability of immune function in the caterpillar Spodoptera littoralis
}

\author{
SC Cotter and K Wilson \\ Institute of Biological Sciences, University of Stirling, Stirling FK9 4LA, UK
}

\begin{abstract}
Phenoloxidase (PO) is believed to be a key mediator of immune function in insects and has been implicated both in non-self recognition and in resistance to a variety of parasites and pathogens, including baculoviruses and parasitoids. Using larvae of the Egyptian cotton leafworm, Spodoptera littoralis, we found that despite its apparent importance, haemolymph PO activity varied markedly between individuals, even amongst insects reared under apparently identical conditions. Sib-analysis methods were used to determine whether individuals varied genetically in their PO activity, and hence in one aspect of immune function. The
\end{abstract}

heritability estimate of haemolymph $\mathrm{PO}$ activity was high $\left(\mathrm{h}^{2}\right.$ $=0.690 \pm 0.069$ ), and PO activity in the haemolymph was strongly correlated with PO activity in both the cuticle and midgut; the sites of entry for most parasites and pathogens. Haemolymph PO activity was also strongly correlated with the degree to which a synthetic parasite (a small piece of nylon monofilament) was encapsulated and melanized ( $r=$ $0.622 \pm 0.142$ ), suggesting that the encapsulation response is also heritable. The mechanism maintaining this genetic variation has yet to be elucidated.

Heredity (2002) 88, 229-234. DOI: 10.1038/sj/hdy/6800031

Keywords: phenoloxidase; encapsulation; immunity; heritability

\section{Introduction}

The invertebrate immune system is significantly simpler than our own in that it lacks an acquired immune response and yet it can cope with a variety of parasites and pathogens. Once recognised as non-self, small invaders may be phagocytosed by circulating haemocytes. Larger organisms are encapsulated, a process by which the parasite is covered by a capsule of haemocytes, which then hardens and melanizes (Gotz, 1986). The haemolymph also contains a variety of antibacterial, antiviral and antifungal proteins. One protein thought to be particularly important in the immune response of insects is phenoloxidase (PO). PO is a copper containing enzyme that catalyses the oxygenation of mono-phenols to 0 diphenols and oxidation of $o$-diphenols to $o$-quinones. These are key steps in the synthesis of melanin, a pigment found in the cuticle (Ashida and Brey, 1995) and around encapsulated foreign bodies (Gotz, 1986). It has been shown in Drosophila that mutants lacking PO are unable to melanize and harden their capsules (Rizki and Rizki, 1990). Other types of phenoloxidase are involved in wound healing and sclerotization of the cuticle (Ashida and Yamazaki, 1990).

Haemolymph PO has been implicated in resistance to a range of pathogens, including nucleopolyhedroviruses (NPVs), fungi, nematodes and parasitoids (Rowley et al, 1990; Ourth and Renis, 1993; Hagen et al, 1994; Hung and Boucias, 1996; Washburn et al, 1996; Bidochka and Hajek,

Correspondence: S Cotter, Institute of Biological Sciences, University of Stirling, Stirling FK9 4LA, UK. E-mail: s.c.cotter@stir.ac.uk

Received 6 November 2000; accepted 7 November 2001
1998; Reeson et al, 1998). However, PO in other parts of the body may also play an important role in immunity. NPVs enter the body via the midgut and proceed by infecting the associated tracheal cells. Washburn et al (1996) showed that in refractory Helicoverpa zea, these infected cells were encapsulated and melanized, halting the spread of the virus. This suggests a possible role for midgut PO in viral resistance. In Anopheles gambiae, Plasmodium cynomolgi ookinetes are encapsulated between the midgut epithelial cells and the midgut basal lamina. It has been shown that refractory individuals have higher midgut PO levels than susceptibles after an infective blood meal. This suggests that their refractoriness may, in part, be due to phenoloxidase activity (Paskewitz et al, 1989).

Active PO in the cuticle may play a role in halting pathogens that enter the haemocoel via that route (eg fungi), by the production of harmful quinones (Chapman, 1998) or by encapsulation of hyphae in the cuticle (Tanada and Kaya, 1993). Despite its apparent importance, there is considerable variation in haemolymph PO activity between individuals in $A$. gambiae (Paskewitz et al, 1989), Spodoptera exigua (Hung and Boucias, 1996) and Lymantria dispar (Bidochka and Hajek, 1998).

PO has also been used as an indicator of immune function. For example, Reeson et al (1998) showed that larvae of the African armyworm, Spodoptera exempta, that had been reared at high densities had significantly higher haemolymph PO levels and higher NPV resistance than those reared solitarily (Reeson et al, 1998). However, as far as we are aware, a direct link between PO activity and intra-specific variation in parasite resistance has yet to be conclusively demonstrated. Thus, as part of an 
ongoing study to examine the association between $\mathrm{PO}$ and pathogen resistance, and to determine any associated costs of pathogen resistance, we have addressed the following questions:

(1) Are PO activity levels in the haemolymph repeatable and heritable?

(2) Is haemolymph $\mathrm{PO}$ activity correlated with $\mathrm{PO}$ activity in different parts of the body, specifically the cuticle and midgut?

(3) Is haemolymph PO activity correlated with the ability to encapsulate parasites in the haemocoel?

\section{Methods}

\section{Spodoptera littoralis culture}

Spodoptera littoralis larvae were collected in Egypt in April 1999 and reared in the laboratory for three generations before experiments were carried out. The stocks were kept at high numbers to avoid inbreeding, with several hundred adults each generation. Larvae were reared at $25^{\circ} \mathrm{C}$ and fed on a semi-artificial wheatgerm-based diet (Reeson, 1999). Experimental larvae were separated into $25 \mathrm{ml}$ polypots at the third instar and assays conducted on newly emerged final instar larvae.

\section{Haemolymph phenoloxidase and protein assays}

Final instar larvae were randomly selected and weighed. A proleg was pierced with a fine, sterile needle and the haemolymph pooled onto parafilm. Eight $\mu$ l of haemolymph were added to $400 \mu \mathrm{l}$ of ice-cold phosphate buffered saline (PBS, pH 7.4; Sambrook et al, 1989) and vortexed. Samples were frozen at $-20^{\circ} \mathrm{C}$ at this point and thawed when all the samples were ready to be measured. Samples were frozen for less than a week in all cases; there is no significant decline in PO activity over this time period $(\mathrm{t}=1.24, \mathrm{df}=58, P=0.22)$. A $100 \mu$ l sample of the haemolymph/PBS mixture was added to $100 \mu \mathrm{l}$ of $20 \mathrm{mM}$ L-Dopa and incubated at $25^{\circ} \mathrm{C}$. After $30 \mathrm{~min}$, the absorbance was measured on a microplate reader at $492 \mathrm{~nm}$. Previous results have shown the reaction to be in the linear phase during this time period (unpublished data). The amount of PO in the sample was calculated in PO units, where one unit is the amount of enzyme required to increase the absorbance by 0.001 per minute. Protein was measured using the BIO-RAD protein assay kit with BSA as the protein standard. Ten $\mu$ l of the haemolymph/PBS mixtures were used to measure the protein in each sample. Absorption was measured on a microplate reader at $600 \mathrm{~nm}$. PO was then expressed as PO units per mg of protein (Hung and Boucias, 1996).

\section{Repeatability of phenoloxidase levels}

Haemolymph was sampled twice from each larva, as described above; the second sample taken either 24 or $48 \mathrm{~h}$ after the first. PO activity was measured in each haemolymph sample and its repeatability determined following the methods of Lessells and Boag (1987).

\section{Midgut and cuticular phenoloxidase assays}

The midgut and cuticle were dissected from each larva and fixed in $2 \%$ formaldehyde and $0.5 \%$ glutaraldehyde in phosphate buffer. After fixation, the cuticles and midguts were washed three times and stored in phos- phate buffer (Wolfgang and Riddiford, 1981). They were then cut in half and each piece weighed. One half of each midgut and cuticle was then placed in $1 \mathrm{ml}$ of $20 \mathrm{mM} \mathrm{L}$ Dopa. The other half was placed in $20 \mathrm{mM}$ L-Dopa saturated with PTU as a control. Samples of $20 \mathrm{mM}$ L-Dopa were incubated along with the experimental samples to control for any spontaneous darkening of the substrate during the experiment. Samples were vortexed and $200 \mu \mathrm{l}$ of the solution pipetted into microtitre plates, absorbance was then measured on a microplate reader at $492 \mathrm{~nm}$ during the linear phase of the reaction. PO activity was expressed as $\mathrm{PO}$ units per gram of cuticle/midgut.

\section{Sib-analysis}

Pupae were sexed and separated into males and females. Ten males were randomly selected and each mated to a virgin female. The females were removed after $24 \mathrm{~h}$ and left in a pot to lay eggs. The males were given further virgin females every day for 5 days. Eggs were collected daily and the caterpillars reared until the third instar. Ten caterpillars were randomly selected from each brood and reared in individual polypots until the final instar. Each caterpillar was weighed and the haemolymph sampled as before and then frozen. When all the samples had been collected they were defrosted and PO and protein were measured as described above, taking three estimates per caterpillar. Variance components were estimated using restricted maximum likelihood (REML) methods in Genstat 5, in which the identity of the sire, dam and individual caterpillar were considered as nested random effects. Heritability estimates were then calculated using the methods of Falconer and Mackay (1996). To ensure robust parameter estimates and standard errors, the variance components procedure was bootstrapped 1000 times.

\section{Encapsulation assay}

Final instar larvae were selected and haemolymph was sampled as described above. A small piece of nylon monofilament $(2.82 \mathrm{~mm} \pm 0.3 \mathrm{~mm})$ was inserted into the haemocoel as an artificial parasite. Twenty-four hours later the larvae were killed, the nylon dissected out and stored in $70 \%$ ethanol. The pieces of nylon were rehydrated, mounted on slides and digitally photographed. The level of encapsulation was scored visually by estimating the amount of cell cover and the level of melanization of each piece of nylon. The scores ranged from 1 to 9 with 1 indicating weak encapsulation and 9 indicating strong encapsulation. The level of melanization and the area of cell cover were also separately quantified using Image Pro-Plus software. Melanization was scored as the mean level of blackness of the nylon (1/Mean Density in Image Pro-Plus) and this was independent of the length of the implant (Major Axis). The area of the attaching cell mass (Area) was also quantified but as this score correlated with nylon length the residuals were used as a corrected score. In order to obtain robust estimates for the correlation coefficients and their standard errors, all correlations were bootstrapped 1000 times.

\section{Results}

Repeatability of haemolymph PO levels

PO activity levels were independent of the larval weight $(r=0.042 \pm 0.088, \mathrm{df}=138, P=0.575)$. There was a sig- 
Table 1 Repeatability of phenoloxidase activity. The within-sample repeatability is a measure of the accuracy of the sampling method. The 24- and 48-h measures indicate the relationship between $\mathrm{PO}$ levels within each larvae from day to day

\begin{tabular}{lccc}
\hline Component & Repeatability \pm SE & $d f$ & $P$ \\
\hline Within sample & $0.983 \pm 0.005$ & 34 & 0.005 \\
Between samples - 24 h & $0.501 \pm 0.120$ & 18 & 0.002 \\
Between samples - 48 h & $0.295 \pm 0.169$ & 34 & 0.032 \\
\hline
\end{tabular}

nificant positive correlation between haemolymph $\mathrm{PO}$ levels measured over $24 \mathrm{~h}$ and $48 \mathrm{~h}$ (Table 1 ). The slopes $(\beta)$ of both lines were significantly different from one and the intercepts $(\alpha)$ were significantly different from zero (24 h: $\beta=0.4563 \pm 0.1666, \mathrm{t}=3.265, \mathrm{df}=18, P=0.0043$; $\alpha=1.944 \pm 0.6746, \mathrm{t}=-2.882, \mathrm{df}=18, P<0.001)(48$ hours: $\beta=0.3453 \pm 0.1811, \mathrm{t}=3.615, \mathrm{df}=34, P<0.001$; $\alpha=2.8123 \pm 0.6302, \mathrm{t}=-4.463, \mathrm{df}=34, P<0.001)$. However, they were not significantly different from each other (comparison of slopes: $\mathrm{t}=0.45, \mathrm{df}=54, P=0.6546$, comparison of intercepts, $\mathrm{t}=-0.941, \mathrm{df}=54, P=0.3511$ ).

\section{Heritability of haemolymph PO levels}

There was significant heritable variation in PO activity (Table 2). The sire and dam heritability estimates were not significantly different (bootstrapped difference between sire and dam estimates $\pm \mathrm{SE}=0.160 \pm 0.230$ ), indicating that there is no significant non-additive or dominance variance (Falconer and Mackay, 1996). Thus, the heritability of PO activity is best estimated by the combined sire + dam estimate, based on the resemblance between full sibs $\left(h^{2}=0.690 \pm 0.069\right)$.

$\mathrm{PO}$ in the haemolymph, midgut and cuticle

There were strong positive correlations between the PO levels in the haemolymph and cuticle $(r=0.590 \pm 0.142$, $\mathrm{df}=17, P=0.005$; Figure 1a), the haemolymph and midgut $(r=0.471 \pm 0.146, \mathrm{df}=17, P=0.020$; Figure $1 \mathrm{~b})$ and the midgut and cuticle $(r=0.701 \pm 0.085, \mathrm{df}=17, P$ $<0.001$; Figure 1c).

\section{Encapsulation assay}

There was a strong, positive correlation between the level of encapsulation of the nylon and the PO activity in the haemolymph using both a qualitative, visual scoring method $(r=0.622 \pm 0.142, \mathrm{df}=15, P=0.005$; Figure 2a) and two quantitative scores. The first, a melanization score, measured the mean level of blackness of the nylon and indicates the amount of melanin deposited on the cell capsule $(r=0.589 \pm 0.149, \mathrm{df}=15, P=0.006$; Figure

Table 2 Heritability of phenoloxidase activity. Variance components were estimated using REML, and heritability estimates, standard errors and 95\% confidence intervals are based on 1000 bootstrapped samples (see text)

\begin{tabular}{lccc}
\hline Component & Heritability $\pm S E$ & $\begin{array}{c}\text { 95\% confidence } \\
\text { interval }\end{array}$ & $P$ \\
\hline sire & $0.770 \pm 0.103$ & $0.577-0.989$ & $<0.001$ \\
dam & $0.610 \pm 0.160$ & $0.300-0.928$ & $<0.001$ \\
sire + dam & $0.690 \pm 0.069$ & $0.561-0.831$ & $<0.001$ \\
\hline
\end{tabular}
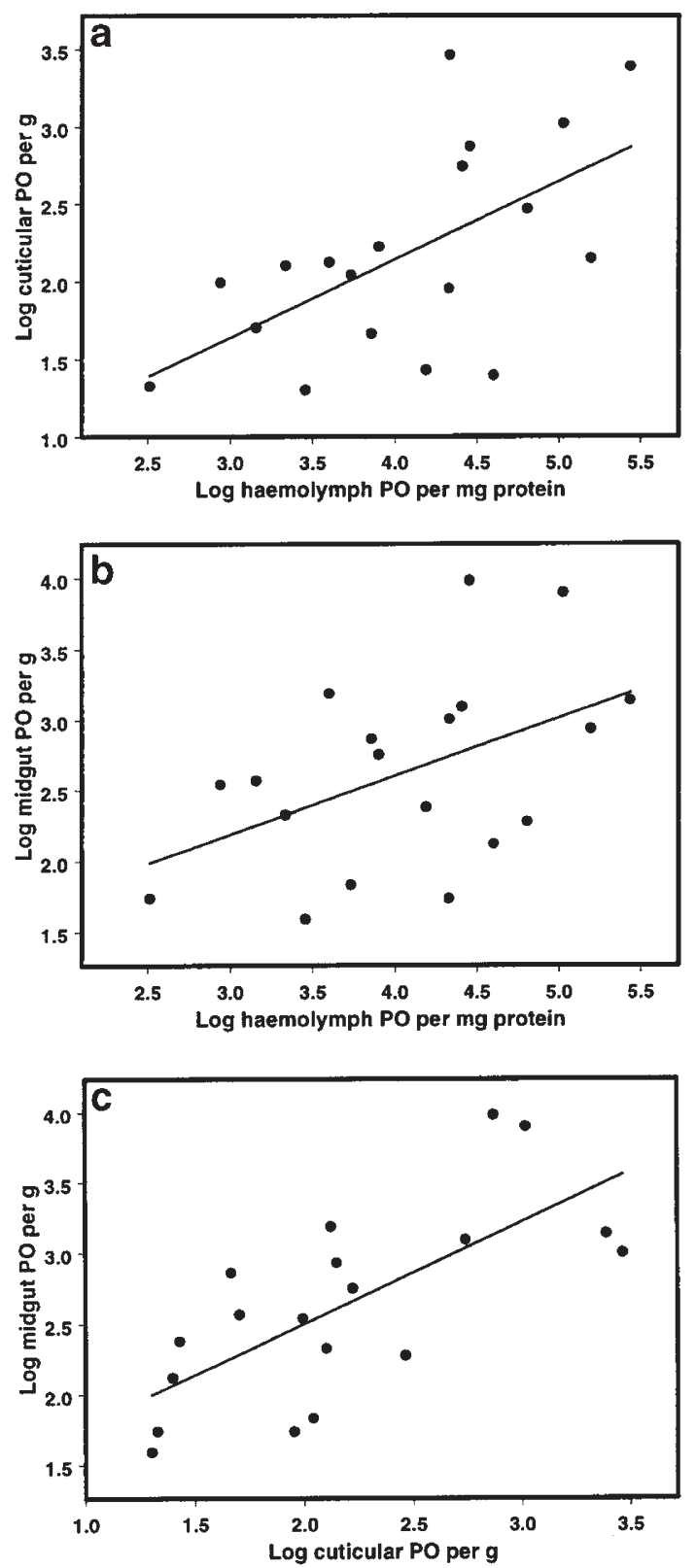

Figure 1 The relationship between phenoloxidase activity in different parts of the body. (a) The correlation between haemolymph PO and cuticular $\mathrm{PO},(\mathbf{b})$ the correlation between haemolymph $\mathrm{PO}$ and midgut $\mathrm{PO}$, (c) the correlation between cuticular $\mathrm{PO}$ and midgut PO.

$2 \mathrm{~b})$, the second scored the volume of the attaching cell mass (capsule area) $(r=0.517 \pm 0.154, \mathrm{df}=15, P=0.041$; Figure 2c). The visual score was strongly correlated with both the melanization score $(r=0.961 \pm 0.018, \mathrm{df}=15, P$ $<0.001)$ and the capsule area $(r=0.615 \pm 0.148, \mathrm{df}=15 ; P$ $=0.014)$. The two quantitative scores were also positively correlated with each other $(r=0.579 \pm 0.144$, df $=15 ; P$ $=0.012$ ).

As the two quantitative encapsulation measurements were highly correlated, the relationship between encapsulation and haemolymph PO was determined using a MANOVA that included both the capsule area and the melanization score as dependent variables. There was a significant effect of haemolymph $\mathrm{PO}$ on the level of 

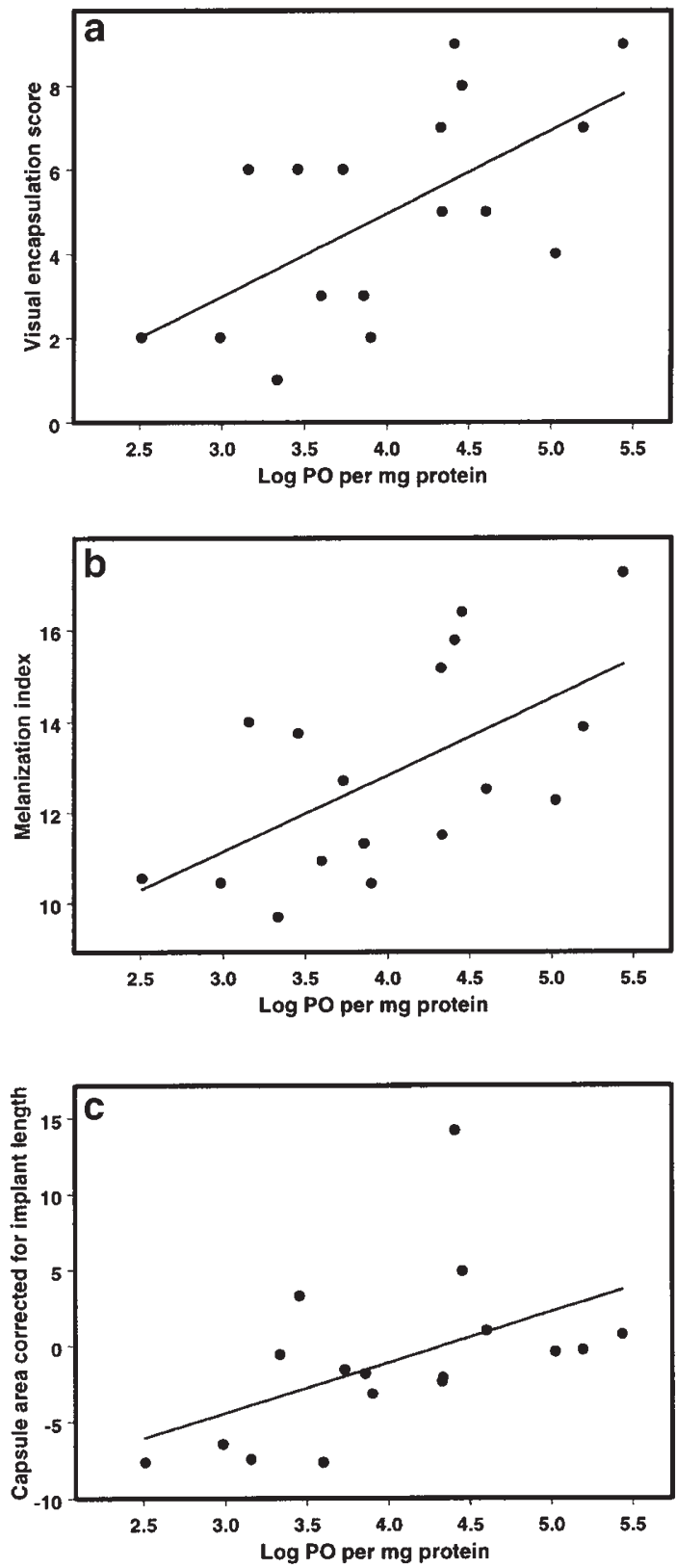

Figure 2 The relationship between haemolymph phenoloxidase levels and the encapsulation of a nylon insert. Encapsulation is measured by (a) visual encapsulation score, (b) degree of melanization of the nylon and (c) capsule area corrected for the length of the nylon.

encapsulation (MANOVA: Wilks' lambda, $\Lambda=0.6019$, approximate $\mathrm{F}_{2,14}=4.63, P=0.029$; univariate tests: capsule area: $\mathrm{F}_{1,15}=5.75, P=0.03$; melanization score: $\mathrm{F}_{1,15}$ $=8.19, P=0.012$ ). Thus, both the capsule area and the melanization score are affected independently by haemolymph PO levels.

\section{Discussion}

PO is not only an important component of the insect immune system but, as shown here, is a repeatable, heritable indicator of an individual's ability to cope with a novel parasite. Although there was significant repeat-

ability of PO levels within individuals both within and across days, the slope of the regression lines comparing PO levels over 24 or 48 h were both significantly different from unity, indicating that PO levels were not the same on each day. This is not particularly surprising as haemocyte number and the levels of certain proteins both increase with age and vary cyclically within instar, peaking just before ecdysis (Chapman, 1998). The larvae used were all in their final instar and so the levels of variation could be due to small differences in the time since the last ecdysis, or the rate at which haemocytes are produced. As the repeatability of the measurement method was high $(r=0.983$, Table 1$)$ very little of the variation was due to within-sample measurement error.

It has been shown in a number of studies that the heritability of traits closely related to fitness tends to be low, as natural selection erodes genetic variation (Gustafsson, 1986; Kruuk et al, 2000; Merila and Sheldon, 2000). It might be expected that heritability estimates from lab populations would be higher than from wild populations due to less variable environmental conditions (Falconer and Mackay, 1996). However, a recent comparative study suggests that there is no significant difference between heritability estimates measured in the laboratory and natural populations (Weigensberg and Roff, 1997). Also, it is important to note that the population used in the current study had been kept in the laboratory for only three generations prior to experimentation, and at numbers high enough to avoid inbreeding. As this is the case, the heritability estimate that we report is likely to be similar to that of the wild population. The high estimate of heritability of haemolymph $\mathrm{PO}$ activity could indicate that this trait is not closely linked to fitness. However, as an unsuccessful response to immune challenge could prove fatal, one would expect important components of the immune system to be strongly tied to individual fitness. So, what could be maintaining the additive genetic variance? One possibility is antagonistic pleiotropy, where a gene confers a positive effect on one component of fitness but a negative effect on another; this is the genetic basis for trade-offs (Roff, 1992). Trade-offs have been shown to occur in a number of studies in which the resistance of a population to a parasite or pathogen has been increased by artificial selection. Boots and Begon (1993) showed that Plodia interpunctella resistant to its granulosis virus had lower egg viability and a longer development time. Kraaijeveld and Godfray (1997) found that Drosophila melanogaster resistant to the parasitoid Asobara tabida had lower larval competitive ability, and similar results have been obtained using the parasitoid Leptopilina boulardi (Fellowes et al, 1998).

Trade-offs could occur between PO and life history traits such as fecundity or longevity, or they could occur within the immune system. In other words, resistance to one parasite or pathogen may occur only at a detriment to another. Aso et al (1985) suggested that haemolymph proPO was a precursor to both haemolymph and cuticular PO. Ashida and Brey (1995) have shown that in Bombyx mori, $\mathrm{PO}$ is synthesised in the haemocytes before being transported to the cuticle. The cuticle and midgut represent important, biologically-active barriers to infection (Paskewitz et al, 1989; Ashida and Brey, 1995). However, with a common precursor, high $\mathrm{PO}$ levels in the cuticle could be bought at the expense of PO in the haemolymph and/or midgut. In this way, resistance to 
fungus and parasitoids that attack via the cuticle could mean lowered levels of resistance to baculoviruses that enter via the midgut. In S. littoralis we have shown that this is not the case. We found no evidence for a trade-off between $\mathrm{PO}$ activity in different body tissues. In fact, there were strong, positive correlations between $\mathrm{PO}$ activity in the haemolymph, midgut and cuticle, indicating that individuals that invest in high levels of haemolymph PO have correspondingly high levels in the midgut and cuticle, suggesting that they could resist attack via either route. A similar pattern has recently been found in the closely related species, Spodoptera exempta (Wilson et al, 2001). Of course, this does not mean that a trade-off between PO levels in different parts of the body does not exist, simply that our experimental protocol was not able to reveal it. Only by manipulating insects so as to change their allocation rules are such trade-offs likely to be revealed (Lessells, 1991). Trade-offs may also occur between functionally different aspects of the insect immune system. For example, it is possible that PO levels may be negatively correlated with the phagocytic activity of haemocytes or the levels of antibacterial proteins or agglutinins in the haemolymph, all of which are important in resistance against bacteria.

Despite all of its suggested roles in the co-ordinated response to immune challenge, there is little evidence linking PO activity in the haemolymph with an individual's ability to resist infection. It has been shown in Anopheles that the ability to encapsulate abiotic material is strongly related to the ability to encapsulate a parasite (Paskewitz and Riehle, 1994; Gorman et al, 1996), and this was the motivation behind our experiment to determine the association between haemolymph PO and the ability to encapsulate a novel pathogen (nylon monofilament). We found that these two measures were strongly correlated, suggesting that individuals that invest in $\mathrm{PO}$ activity in the haemolymph, midgut and cuticle have a greater capacity for encapsulating parasites. Future studies will examine any costs associated with increased levels of PO and the association between PO and resistance to entomopathogens.

\section{Acknowledgements}

We would like to thank Jenny Cory, Rosie Hails, Esmat Hegazi, Loeske Kruuk, Andy Reeson, Margaret MacKinnon and Sue Smith for practical assistance and for helpful comments on the paper. This study was supported by the Natural Environment Research Council and CEH-Oxford and was written whilst KW was in receipt of a NERC Advanced Research Fellowship.

\section{References}

Ashida M, Brey PT (1995). Role of the integument in insect defense: prophenoloxidase cascade in the cuticular matrix. Proceed Nat Acad Sci USA Immunol 92: 10698-10702.

Ashida M, Yamazaki HI (1990). Biochemistry of the phenoloxidase system in insects: with special reference to its activation. In: Ohnishi E, Ishizaki H (eds) Moulting and Metamorphosis, Springer Verlag. pp 239-265.

Aso Y, Kramer KJ, Hopkins TL, Lookhart GL (1985). Characterization of hemolymph protyrosinase and a cuticular activator from Manduca-Sexta (L). Insect Biochem 15: 9-17.

Bidochka MJ, Hajek AE (1998). A nonpermissive entomophthor- alean fungal infection increases activation of insect prophenoloxidase. J Invert Pathol 72: 231-238.

Boots M, Begon M (1993). Trade-offs with resistance to a granulosis-virus in the Indian meal moth, examined by a laboratory evolution experiment. Funct Ecol 7: 528-534.

Chapman RF (1998). The Insects; Structure and Function, 4th Edition. Cambridge University Press: UK.

Falconer DS, Mackay TFC (1996). Introduction to Quantitative Genetics, 4th edition. Longman Group Ltd.

Fellowes MDE, Kraaijeveld AR, Godfray HCJ (1998). Trade-offs associated with selection for increased ability to resist parasitoid attack in Drosophila melanogaster. Proc Roy Soc Lond B 265: 1553-1558.

Gorman MJ, Cornel AJ, Collins FH, Paskewitz SM (1996). A shared genetic mechanism for melanotic encapsulation of CM-sephadex beads and the malaria parasite, Plasmodium cynomolgi B, in the mosquito Anopheles gambiae. Exper Parasitol 84: 380-386.

Gotz P (1986). Encapsulation in arthropods. In: Brehelin M (eds) Immunity in Invertebrates - Cells, Molecules and Defense Reactions, Springer Verlag.

Gustafsson L (1986). Lifetime reproductive success and heritability - empirical support for Fisher Fundamental Theorem. Am Nat 128: 761-764.

Hagen HE, Grunewald J, Ham PJ (1994). Induction of the prophenoloxidase-activating system of Simulium (Diptera, Simuliidae) following Onchocerca (Nematoda, Filarioidea) infection. Parasitology 109: 649-655.

Hung SY, Boucias DG (1996). Phenoloxidase activity in the hemolymph of naive and Beauvaria bassiana-infected Spodoptera exigua larvae. J Invert Pathol 67: 35-40.

Kraaijeveld AR, Godfray HCJ (1997). Trade-off between parasitoid resistance and larval competetive ability in Drosophila melanogaster. Nature 389: 278-280.

Kruuk LEB, Clutton-Brock TH, Slate J, Pemberton JM, Brotherstone S, Guinness FE (2000). Heritability of fitness in a wild mammal population. Proc Nat Acad Sci USA 97: 698-703.

Lessells CM, Boag PT (1987). Unrepeatable repeatabilities: a common mistake. The Auk 104: 116-121.

Lessells CM (1991). The evolution of life histories. In: Davies NB (eds) Behavioural Ecology - An Evolutionary Approach, 3rd edn. Blackwell Scientific Publications.

Merila J, Sheldon BC (2000). Lifetime reproductive success and heritability in nature. Am Nat 155: 301-310.

Ourth DD, Renis HE (1993). Antiviral melanisation reaction of Heliothis virescens haemolymph against DNA and RNA viruses in vitro. Comp Biochem Physiol 105B: 719-723.

Paskewitz S, Riehle MA (1994). Response of Plasmodium refractory and susceptible strains of Anopheles gambiae to inoculated sephadex beads. Dev Comp Immunol 18: 369-375.

Paskewitz SM, Brown MR, Collins EH, Olea A (1989). Ultrastructural localisation of phenoloxidase in the midgut of refractory Anopheles gambiae and association of the enzyme with encapsulated Plasmodium cynomolgi. J Parasitol 75: 594600.

Reeson AF (1999). Density dependent phase polyphenism in the African armyworm Spodoptera exempta (Lepidoptera: noctuidae). PhD Thesis, University of Southampton.

Reeson AF, Wilson K, Gunn A, Hails RS, Goulson D (1998). Baculovirus resistance in the noctuid Spodoptera exempta is phenotypically plastic and responds to population density. Proc Roy Soc Lond B-Biolog Sci 265: 1407, 1787-1791.

Rizki RM, Rizki TM (1990). Encapsulation of parasitoid eggs in phenoloxidase-deficient mutants of Drosophila melanogaster. J Insect Physiol 36: 523-529.

Roff DA (1992). The Evolution of Life Histories - Theories and Analysis, Chapman \& Hall.

Rowley AF, Brookman JL, Ratcliffe NA (1990). Possible involvement of the prophenoloxidase system of the locust, Locusta migratoria, in antimicrobial activity. J Invert Pathol 56: 31-38.

Sambrook J, Fritsch EF, Maniatis T (1989). Molecular Cloning: A 
Laboratory Manual, 2nd edition. Cold Spring Harbor Laboratory Press.

Tanada Y, Kaya HK (1993). Insect Pathology, Academic Press.

Washburn JO, Kirkpatrick BA, Volkman LE (1996). Insect protection against viruses. Nature 383: 767.

Weigensberg I, Roff DA (1997). Natural heritabilities: can they be reliably estimated in the laboratory? Evolution 50: 21492157.
Wilson K, Cotter SC, Reeson AF, Pell JK (2001). Melanism and disease resistance in insects. Ecol Lett 4: 637-649.

Wolfgang WJ, Riddiford LM (1981). Cuticular morphogenesis during continuous growth of the final instar larva of a moth. Tissue $\mathcal{E}$ Cell 13: 757-772. 